\title{
Excited Ions in Intense Femtosecond Laser Pulses: Laser-Induced Recombination
}

\author{
I. D. Williams, ${ }^{1, *}$ J. McKenna, ${ }^{1}$ J. Wood, ${ }^{2}$ M. Suresh, ${ }^{1}$ W. A. Bryan, ${ }^{2,3}$ S. L. Stebbings, ${ }^{2}$ E. M. L. English, ${ }^{2}$ C. R. Calvert, ${ }^{1}$ \\ B. Srigengan, ${ }^{1}$ E. J. Divall, ${ }^{3}$ C. J. Hooker, ${ }^{3}$ A. J. Langley, ${ }^{3}$ and W. R. Newell ${ }^{2,}{ }^{\dagger}$ \\ ${ }^{1}$ Department of Physics, Queen's University Belfast, Belfast BT7 1NN, United Kingdom \\ ${ }^{2}$ Department of Physics and Astronomy, University College London, London, WC1E 6BT, United Kingdom \\ ${ }^{3}$ Central Laser Facility, Rutherford Appleton Laboratory, Chilton, Didcot, Oxon., OX11 OQX, United Kingdom
}

(Received 3 July 2007; published 26 October 2007)

\begin{abstract}
Electron-ion recombination in a laser-induced electron recollision is of fundamental importance as the underlying mechanism responsible for the generation of high-harmonic radiation and hence for the production of attosecond pulse trains in the extreme ultraviolet and soft x-ray spectral regions. By using an ion beam target, remotely prepared to be partially in long-lived excited states, the recombination process has for the first time been directly observed and studied.
\end{abstract}

Intense ultrashort laser pulses are an important tool for probing the nonlinear physics of atoms, molecules and clusters in the inherent strong fields. Such studies have been used to elucidate fundamental quantum processes in the regime where electric $(E)$ field strengths are of a similar order of magnitude to that binding electrons and where pulse time scales approaching a femtosecond constrain the quantum dynamics. In addition some of the resulting processes are being harnessed, for example, high-harmonic generation to produce attosecond $\mathrm{x}$-ray pulses [1] in order to further probe atomic systems [2] and for practical nanotechnological applications $[3,4]$.

Arguably the single most important aspect of intense field studies is the "rescattering" of an ionized electron from its ionic core in a linearly polarized pulse. Proposed in a seminal paper by Corkum [5], the process relies on the ejected electron periodically revisiting the core as it is driven to and from it by the oscillating $E$-field of the laser. Acceleration in the external field, coupled with Coulombic acceleration and focussing may lead to recollision with the ion core. The narrow momentum distribution of the wave packet results in a significant probability of electron-ion interaction. This opens up the fascinating prospect of studying electron-ion scattering phenomena, notoriously difficult to investigate in a conventional beam-beam approach [6], with the added bonus that the electron "pulse" is on a femtosecond time scale [7].

The possible electron collision mechanisms, i.e., elastic scattering, inelastic scattering, and recombination, have all been observed by various means. Elastic scattering leads to momentum transfer, such that the scattered electron is unlikely to further recollide with the core and will be observable at the high energy extreme of the detected electron spectrum, contributing to the above-threshold ionization process [8]. Inelastic scattering contributes to the nonsequential ionization process, either directly by electron impact ionization [9] or indirectly by excitation followed by tunnelling ionization in the field [10]. Recombination, which may be regarded as the inverse of above-threshold ionization, has been observed by detection of the stabilizing photon, the source of high-harmonic generation [11]. However, the underlying effect of laserinduced recombination has not been independently experimentally studied in the femtosecond pulse regime, and indeed recombination has never been detected in the product ion channel. It is particle detection of this effect that forms the focus of this Letter.

A well established technique for isolating recollision events compares ionization signal from atomic targets produced by linear and circular polarized femtosecond laser pulses. With circularly polarized pulses, ionized electrons have large transverse momenta and hence a negligible probability of recollision. Thus, inelastic rescattering effects are seen as an enhancement to the linear double ionization signal [12] or, by conservation of flux, a corresponding decrease in the linear single ionization signal [13]. It might be expected that recombination could similarly be observed as a loss of flux in the single ionization linear signal when compared to the corresponding circular signal. However, the difficulty with this approach to studying recombination via particle detection lies in the fact that if the field is sufficiently intense to ionize an electron from a ground state atom in the first place, then recombination to any bound atomic state will usually be rapidly followed by reionization. Such a scenario has recently been described by $\mathrm{Hu}$ and Collins [14].

One solution would be to have a target of excited atoms, which will ionize at a lower intensity than the ground state; hence, recombination into the ground state would lead to a stable product in a field too weak for subsequent reionization. In a previous communication, we described how a fast atomic ion beam, $\mathrm{Ar}^{+}$, produced by electron impact in an ion source was accelerated and transported to an interaction region where it formed the target for intense femtosecond laser pulses [15]. Therein, we demonstrated the suppression of nonsequential ionization in multiple ionization of ground state $\mathrm{Ar}^{+}$compared to that for neutral Ar. We also noted the fact that $\mathrm{Ar}^{2+}$ ionization signal was 
detected not only from the ground state but also from an ensemble of metastable excited states. In the present Letter, we report on how $\mathrm{Kr}^{+}$ions prepared in such an excited state allowed direct observation of laser-induced recombination via particle detection.

$\mathrm{Kr}$ gas was ionized by electron impact in a plasma discharge source. Due to the high electron temperatures sustained in the source, the $\mathrm{Kr}^{+}$ions were formed in a broad range of excited states, most of which decayed within the source itself. Ions were extracted into a beam, electrostatically focussed and momentum analyzed, so that a $2 \mathrm{keV}$ beam of pure $\mathrm{Kr}^{+}$could be transported into the interaction region. The flight time of microseconds ensured that only ions in the ground state and long-lived metastable states were present at the point of interaction with the laser pulses. Ionization products, $\mathrm{Kr}^{q+}(q>1)$, were energy analyzed in a parallel plate analyzer before being detected, with time-of-flight information also recorded to assist signal analysis via a fast digital oscilloscope.

The Astra laser system at the CCLRC Rutherford Appleton Laboratory (UK) consisted of a Ti:Sapphire laser (790 nm) utilizing chirped pulse amplification with $40 \mathrm{fs}$ pulse duration, $10 \mathrm{~Hz}$ repetition rate, and energy of $20 \mathrm{~mJ}$ per pulse. The beam of diameter $22 \mathrm{~mm}$ was focused into the interaction region via a $250 \mathrm{~mm}$ focal length lens. A half-wave plate in combination with a linear polarizer was used to attenuate the laser energy to suit the experimental requirements, and a quarter-wave plate was used to change linear polarization to circular polarization or vice-versa. Pulse energy was monitored throughout with shot-to-shot fluctuations discriminated at a $10 \%$ level.

Ionization signal was measured as a function of pulse intensity using a restricted volume, intensity selective scan (ISS), technique [16]. Here, the final focusing lens is translated ( $z$-value) so that the ion beam target interacts with regions of differing intensity within the focussed laser beam. This has the distinct advantage of increasing the interaction volume as the intensity decreases, thus enhancing the sensitivity to the low intensity processes. Additionally, the finite diameter of the ion beam (300 $\mu \mathrm{m}$ FWHM) reduces the range of intensities experienced in a single measurement. At each $z$-value, a measurement was taken with both linear and circular polarized pulses. The resulting comparison permitted electron rescattering information to be deduced.

For a valid comparison to be made, there first had to be a normalization of the signals expected from "pure" sequential ionization for both linear $\left(S_{L}\right)$ and circular $\left(S_{C}\right)$ cases. We have described elsewhere [13] how in a detailed study of the single and double ionization of $\mathrm{Ne}$ (where electron rescattering effects are small), a normalization factor of 0.65 was accurately determined for the linear to circular ratio. This factor is the necessary attenuation of the incident linear beam compared to that used pre-quarter wave plate for production of the circular polarized pulses. This differs to the intuitive factor of 0.5 which would give equivalent peak $E$-fields in both cases. One can understand qualitatively the reason for this difference as arising due to the constant nature of the magnitude of the $E$-vector in a circular pulse while it will oscillate between zero and the peak in a linear pulse. However, to also quantify the effect, shown in the left panel of Fig. 1(a) are the predictions of Perelomov-Popov-Terent'ev (PPT) theory [17] for the ionization of excited $\mathrm{Kr}^{+}$ions with an ionization potential of $8.3 \mathrm{eV}$. This choice of state allows the intensity range to span both the multiphoton (MPI) and tunnelling (TI) regimes as defined by the Keldysh adiabaticity parameter, $\gamma$, indicated on the figure (MPI: $\gamma \gg 1$, TI: $\gamma<1$ ). Clearly
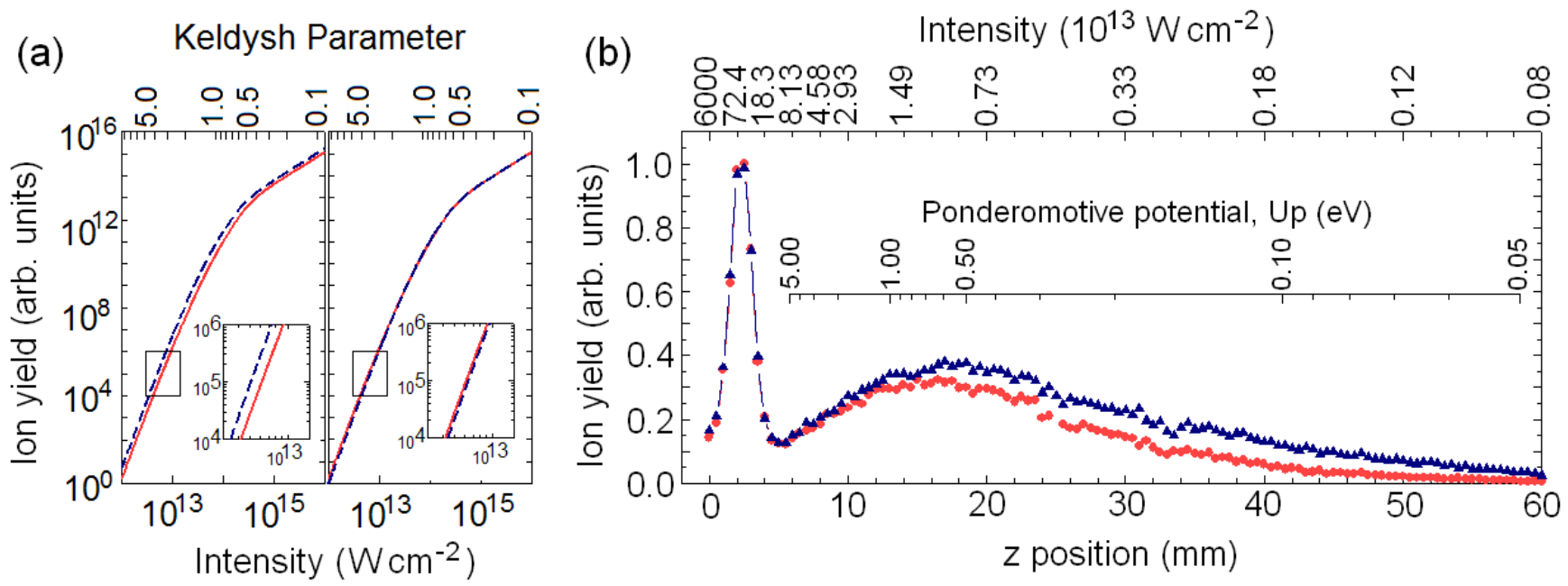

FIG. 1 (color online). (a) Predictions of PPT theory for the multiphoton-tunnelling single ionization of excited (ionization potential $=8.3 \mathrm{eV}$ ) $\mathrm{Kr}^{+}$ions: left panel, unshifted linear (solid curve) and circular (broken curve) yields; right panel, circular yield shifted up in intensity by a factor of 1.3 - equivalent to using a linear to circular intensity normalization ratio of 0.65 [0.5 $\times 1.3$ ] as described in the text. (b) ISS signal measured as a function of focusing lens position, with respect to ion beam axis, for linear (circles) and circular (triangles) polarizations for $\mathrm{Kr}^{2+}$ production from the target beam of $\mathrm{Kr}^{+}$ions. 
the circular rate exceeds the linear rate for equivalent peak $E$-field. Following normalization of the intensity by our experimentally determined matching factor of 0.65 (by shifting the circular curve up in intensity by a factor of $1.3[0.5 \times 1.3=0.65])$, right panel, excellent agreement is observed over a broad range of intensity.

Experimental measurements of $S_{L}$ and $S_{C}$ for $\mathrm{Kr}^{2+}$ production from ionization of a $\mathrm{Kr}^{+}$target are presented in Fig. 1(b). Signal strength is plotted as a function of $z$-value as the lens is translated from $z=0$, corresponding to a maximum intensity of $6 \times 10^{16} \mathrm{~W} \mathrm{~cm}^{-2}$ at the focus using linearly polarized light, to $z=60 \mathrm{~mm}$ corresponding to an intensity of $8 \times 10^{11} \mathrm{~W} \mathrm{~cm}^{-2}$. A guide to the nonlinearity in intensity variation over the $z$-translation is also given in the figure.

The narrow peak observed in the range $z=0-4 \mathrm{~mm}$ is due to ionization of the $4 s^{2} 4 p^{5}{ }^{2} P_{3 / 2}^{0}$ ground state of $\mathrm{Kr}^{+}$, with an ionization potential of $24.6 \mathrm{eV}$. Of primary importance here is the nearly identical peak shapes for ground state ionization signal from linear and circular pulses. There is little evidence of any loss of flux from the linear channel which might appear as an enhancement due to inelastic rescattering in the double ionization signal. In fact, we have also measured the $\mathrm{Kr}^{3+}$ product signal confirming this, the result being in agreement with our previous assertion that nonsequential multiple ionization appears to be suppressed when an ionic target replaces the respective isonuclear neutral target [15]. More importantly for the present study, the equivalence of shape of $S_{L}$ and $S_{C}$ shows a ground state atomic ion target behaving in identical fashion to a neutral atom target under almost "pure" sequential ionization conditions. The calibration of the 0.65 normalization factor was carried out in parallel using a $\mathrm{Ne}$ gas target in a different spectrometer and vacuum system under identical laser conditions.

The broad peak tailing out to lower intensities at high $z$-value is due to a combination of multiphoton and tunnelling ionization of an ensemble of metastable states of $\mathrm{Kr}^{+}$, and is the signal of primary concern in the present study. The possible contributing states are $4 s^{2} 4 p^{4}\left({ }^{3} P\right) 4 d^{4} D_{7 / 2}$, ${ }^{4} F_{9 / 2},{ }^{4} F_{7 / 2}$, and ${ }^{2} F_{7 / 2}$ lying at $14.9,15.6,15.9$, and $16.3 \mathrm{eV}$, respectively, above the ground state [18]. These states are all sufficiently long-lived ( $>1$ second), by virtue of the $\Delta J$ selection rule to the ground state and Laporte's rule to lower lying excited states, that they suffer negligible depletion during transportation from the source to the point of interaction. The significantly lower ionization potentials for these states means that ionization proceeds at much lower intensities than for the ground state, resulting in well resolved ground and metastable signal peaks as seen in Fig. 1(b). By comparing simulated fits to the circular metastable and ground state peaks using a volume fitting procedure [19], and correcting for volume enhancement, a metastable fraction of $0.015 \pm 0.010$ for the sum of the metastable states has been measured. This is consistent with previous measurements [20] from comparable ion sources. However, the relative populations of the unresolved individual excited states are unknown. It should be noted that the good signal to noise ratio obtained from the small fraction of excited states present was due to the volume enhancement achievable with the ISS technique.

It is immediately apparent from Fig. 1(b) that while there is close overlap between $S_{L}$ and $S_{C}$ for the ground state peak, the metastable linear signal is lower than the corresponding circular signal over most of the intensity range. This is exactly the result being sought to show evidence of recombination in the electron recollision with the core. At pulse intensities well below the observable threshold for ground state ionization, recombination into the ground state results in a stable $\mathrm{Kr}^{+}$ion, thus reducing flux from the $\mathrm{Kr}^{2+}$ product signal.

That the difference could be due to a breakdown in the validity of the normalization factor of 0.65 for an excited state seems highly unlikely, as evidenced by the PPT calculation. Indeed, the small difference in the normalized PPT signals at the lowest intensities in Fig. 1(a) (right panel) shows the reverse trend to our observation with the linear signal exceeding the circular. As a further test however, the normalization factor was varied in an attempt to match the linear and circular curves over the metastable peaks. This was found to be impossible as matching at the low $z$-value extremity resulted in a poor match at the high $z$-values and vice-versa; i.e., the intensity dependence of $S_{L}$ and $S_{C}$ differed regardless of the normalization factor used.

In previous work with ground state noble gas atoms (e.g., [13]) loss of linear flux has been observed in single ionization measurements due to inelastic rescattering converting singly charged to multiply charged ions. This "missing" flux then reappears at the corresponding $z$-values in the double ionization linear signal. That this cannot be happening here is evident from a consideration of the ponderomotive potential $\left(U_{P}\right)$ [21] values also shown on Fig. 1(b). There is clearly insufficient recollison energy (maximum $3.17 U_{P}$ ) for the ejected electron to be inelastically scattered given that the resonance transition lies at $13.5 \mathrm{eV}$ above the ground state. In confirmation of this, no signal was experimentally discernible in the $\mathrm{Kr}^{3+}$ spectrum over the range of $z$-values where the metastable peaks are observed.

To confirm the hypothesis that the loss of metastable linear flux is due to recombination of the electron with the $\mathrm{Kr}^{2+}$ core, the normalized signal difference $\left(S_{C}-S_{L}\right) / S_{C}$ has been plotted against maximum electron return energy in Fig. 2. By taking this ratio of recombination to initial ionization signal strengths, we obtain the probability for recombination of ionized electrons. The absolute scale for the probability is shown on the right axis of the figure. Also plotted is the radiative electron capture cross-section, estimated through generalization of the well-known Kramers formula, for a Coulomb potential, by introduction of an effective ionic charge $Z_{\text {eff }}$ [22], 


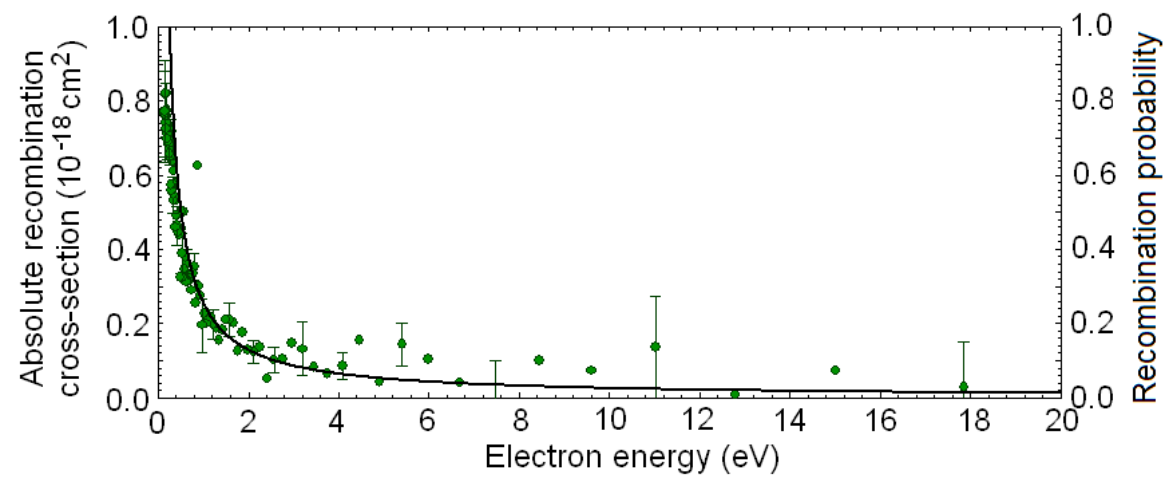

FIG. 2 (color online). Plot showing the experimental recombination probability (right axis) normalized for the initial ionization events $\left[\left(S_{C}-S_{L}\right) / S_{C}\right]$ (circles) as a function of the maximum electron return energy $\left(3.17 U_{P}\right)$. Also plotted (solid curve) is the absolute recombination cross-section (left axis) determined using the Kramers formula. Error bars depict statistical uncertainty at $1 \sigma$.

$$
\sigma_{n}=\frac{32 \pi}{3 \sqrt{3} c^{3}} \frac{\left(0.5 Z_{\mathrm{eff}}^{2}\right)^{2}}{n \varepsilon\left(0.5 Z_{\mathrm{eff}}^{2}+n^{2} \varepsilon\right)} .
$$

In this formula, $\varepsilon$ is the initial electron energy, $n$ is the principal quantum number of the final electron state, and atomic units are used. The definition of $Z_{\text {eff }}$ used here is $Z_{\text {eff }}=0.5\left(Z+Z_{i}\right)$, where $Z$ is the ionic charge of the bare (fully striped) nucleus and $Z_{i}$ is the charge of the ion to which the electron will recombine. Thus, for the fit, parameters $\left(Z, Z_{i}\right)=(36,2)$ and $n=4$ were used, as recombination of the electron is back into the $\mathrm{Kr}^{2+} 4 s^{2} 4 p^{4}$ core. However, it is known [22] that $Z_{\text {eff }}$ is a compromise value, and as such the formula is expected to overestimate the cross-section at low energies while underestimating at high energies, i.e., the effective charge seen by the recombining electron increases with its kinetic energy. We note that the cross-section is plotted as an absolute value in units of $\mathrm{cm}^{2}$ indicated by the scale on the left axis of the figure. The experimental data present a relative cross-section measurement.

The good agreement observed with the cross-section fit provides strong confirmation that recombination is directly responsible for the $S_{L}$ and $S_{C}$ differences observed in Fig. 1(b). Indeed, it demonstrates that the process is similar in nature to that of a classical free electron incident on an ion core. This is an interesting finding as it highlights a novel method of performing in situ studies of electron-ion recombination processes, where the electron already possesses a strong correlation with the target, and in addition, the scattering energy can be controlled by the ponderomotive energy in the drive laser pulse. Furthermore, the good agreement with theory, based upon field-free recombination, suggests that the influence of the laser pulse at the time of recollision plays a negligible role. This should not be considered surprising given that the optimum time for recollision, and thus recombination, is at a phase of the laser field when the $E$ vector can be considered as small. However, confirmation of this fact, which is still being sought, is important for certain applications; e.g., by neglecting the field influence, high-harmonic generation yield calculations can more readily be extended to larger molecular systems [23]. Finally, this fundamental investigation of the process responsible for high-harmonic atto- second pulse generation (e.g., [24]) introduces a new perspective to the study of the underlying physics.

The experiments were undertaken with the financial support of the Engineering and Physical Sciences Research Council (UK). J.M. K. and C. R. C. wish to acknowledge funding from the Department for Employment and Learning (NI); M. S. from the International Research Centre for Experimental Physics at QUB; J. W., E. M. L. E., and S. L. S. from ESPRC.

*i.williams@qub.ac.uk

†w.r.newell@ucl.ac.uk

[1] M. Drescher et al., Science 291, 1923 (2001).

[2] F. Krausz, Phys. World 14, 41 (2001).

[3] M. Weiland et al., Ultramicroscopy 102, 93 (2005).

[4] A.R. Libertun et al., CLEO, Trends in Optics and Photonics Series Vol. 96 (Optical Society of America, Washington, DC, 2004), article JMD4.

[5] P. B. Corkum, Phys. Rev. Lett. 71, 1994 (1993).

[6] I. D. Williams, Rep. Prog. Phys. 62, 1431 (1999).

[7] H. Niikura et al., Nature (London) 417, 917 (2002).

[8] G. G. Paulus et al., Phys. Rev. Lett. 72, 2851 (1994).

[9] R. Moshammer et al., Phys. Rev. A 65, 035401 (2002).

[10] B. Feuerstein et al., Phys. Rev. Lett. 87, 043003 (2001).

[11] A. McPherson et al., J. Opt. Soc. Am. B 4, 595 (1987).

[12] B. Walker et al., Phys. Rev. A 48, R894 (1993).

[13] M. Suresh et al., Nucl. Instrum. Methods Phys. Res., Sect. B 235, 216 (2005).

[14] S.X. Hu and L.A. Collins, Phys. Rev. A 70, 013407 (2004).

[15] J. B. Greenwood et al., Phys. Rev. Lett. 88, 233001 (2002).

[16] P. Hansch and L. D. Van Woerkom, Opt. Lett. 21, 1286 (1996).

[17] A. M. Perelomov, V. S. Popov, and M. V. Terent'ev, Sov. Phys. JETP 23, 924 (1966).

[18] H. D. Hagstrum, Phys. Rev. 104, 309 (1956).

[19] A. A. A. El-Zein et al., Phys. Scr. T92, 119 (2001).

[20] P. Varga and H. Winter, Phys. Rev. A 18, 2453 (1978).

[21] The ponderomotive energy, $U_{P}$, is the cycle-averaged oscillation energy of a free electron in the laser field and is proportional to laser intensity and carrier wavelength.

[22] Y. S. Kim and R. H. Pratt, Phys. Rev. A 27, 2913 (1983).

[23] B. Zimmermann, M. Lein, and J. M. Rost, Phys. Rev. A 71, 033401 (2005).

[24] M. Hentschel et al., Nature (London) 414, 509 (2001). 